Please do not remove this page

RMIT

UNIVERSITY

\title{
Search for high-performance probe-fed stacked patches using optimization
}

Mitchell, Arnan; Lech, Margaret; Kokotoff, D; Waterhouse, Rod

https://researchrepository.rmit.edu.au/esploro/outputs/9921858992501341/filesAndLinks?institution=61RMIT_INST\&index=null

Mitchell, A., Lech, M., Kokotoff, D., \& Waterhouse, R. (2003). Search for high-performance probe-fed stacked patches using optimization. IEEE Transactions on Antennas and Propagation, 51(2), 249-255. https://doi.org/10.1109/TAP.2003.809070

Published Version: https://doi.org/10.1109/TAP.2003.809070

Repository homepage: https://researchrepository.rmit.edu.au

(c) 2003 IEEE. Personal use of this material is permitted. However, permission to reprint/republish this material for advertising or promotional purposes or for creating new collective works for resale or redistribution to servers or lists, or to reuse any copyrighted component of this work in other works must be obtained from the IEEE.

Downloaded On 2023/04/26 19:00:14 +1000 


\title{
Search for High-Performance Probe-Fed Stacked Patches Using Optimization
}

\author{
Arnan Mitchell, Margaret Lech, David M. Kokotoff, and Rod B. Waterhouse, Senior Member, IEEE
}

\begin{abstract}
High-performance circular probe-fed stacked patch antenna designs are explored through the use of numerical optimization. New trends are sought to aid understanding and to suggest novel solutions. We describe the optimization technique, present a new design trend relating efficiency and bandwidth to the choice of substrate dielectric, and propose and demonstrate a novel, optimized antenna achieving $33 \%$ bandwidth whilst maintaining greater than $80 \%$ surface wave efficiency.
\end{abstract}

Index Terms-Microstrip antennas, optimization methods, surface waves.

\section{INTRODUCTION}

$\mathbf{M}$ ICROSTRIP patch antennas are universally accepted as one of the most versatile style of antenna structures. Over the years, inherent problems associated with these radiators have been addressed producing patches with enhanced impedance bandwidth [1]-[4], improved efficiency [5]-[7] and even reduced conductor size [8]-[10]. Due to this global research effort, variants of the microstrip patch are now incorporated in applications ranging from mobile base-station antennas [11] to handset terminals [12]. Despite the current level of design sophistication, there are still many areas of patch antenna technology that warrant continued research to more fully understand the factors determining their performance.

Probably the most fundamental parameter of a microstrip patch antenna is the choice of dielectric laminate used under the conductor. Preliminary investigations have been undertaken to examine the overall effect of the dielectric constant and thickness of the materials for both single layered and stacked patch configurations on the impedance bandwidth and radiation efficiency of the antenna [13]. In [13], the authors derived a "figure of merit" using a simplified theoretical approach to compare the relative merits of different substrates. Although the results presented for single layered configurations followed well-established trends [14], the stacked patch configurations confirmed the somewhat surprising results presented in [7], namely, that it was not necessary to use low dielectric constant materials for both laminates to yield good impedance bandwidth and efficiency. These findings are contradictory to previously derived 'rules of thumb' and logically lead to the following question: "what composition of materials can give the optimum impedance bandwidth and antenna efficiency?"

Manuscript received May 4, 2001.

A. Mitchell and M. Lech are with the The Royal Melbourne Institute of Technology (RMIT), Melbourne, VIC, Australia.

D. M. Kokotoff is with Gabriel Electronics Inc., Scarborough, ME 04070 USA.

R. B. Waterhouse is with Dorsal Networks, Inc., Columbia, MD 21046 USA Digital Object Identifier 10.1109/TAP.2003.809070
In this paper, we explore material combinations to obtain the optimum impedance and efficiency performance for a probe-fed stacked microstrip patch antenna. To search for these optimal solutions manually would require many hours of a skilled antenna engineer's time and would likely be colored by the rules of thumb discovered previously. We instead employ numerical optimization [15] to automatically synthesize high-performance antenna geometries under specified conditions and within given constraints. Using this approach, the dielectric material values can be set and optimal antennas using these materials can be synthesized. By comparing the performance of the solutions resulting from different material combinations, design trends suggesting the best material combinations for efficient broad-band patch antennas may be identified.

It will be shown that the lower laminate in a stacked patch configuration should have a dielectric constant of approximately 5 and the value for the higher layer should have a relative dielectric constant close to unity to give the best impedance bandwidth without compromising the antenna efficiency. Design trends and experimental verification of the theoretical findings is presented herein.

\section{OPTIMIZATION APPROACH}

Numerical techniques have been used previously in the investigation of optimum microstrip patch antennas. Recently an investigation was conducted on the impact of the conductor shape of a microstrip patch antenna on the bandwidth of the antenna and how it can be optimized to yield performance compared to more conventional, canonical patch geometries [16]. Here, a genetic algorithm was utilized to search for conductor shapes that could improve the impedance response. Bandwidths on the order of $20 \%$ were achieved. Conversely, the approach used here maintains the same basic geometry, the direct contact probe fed circular stacked patch antenna, but varies the parameters of this geometry. A report of early work on this optimization approach can be found in [15].

Fig. 1 presents a diagram of the optimization procedure used. It begins with a random set of antenna parameters. It would be possible to begin with a known solution, but so as not to bias the solution we begin with purely random parameters. These are then checked to see if they fall within loose constraints, failure results in a penalty being applied to the cost function. If the parameters are within constraints, the antenna response is modeled and the results are assessed using a cost function. The result of this cost function is compared to the cost function of the previous iteration to determine if the solutions has converged. If the normalized change in cost is smaller than a specified tolerance, then the solution is deemed converged and the optimization is 


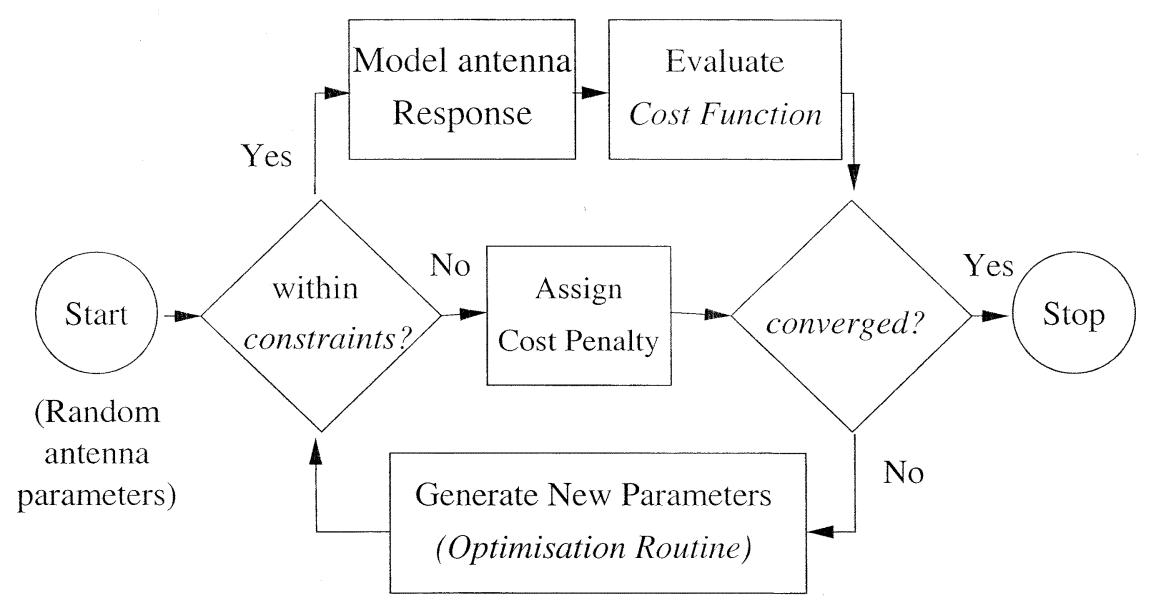

Fig. 1. Flow chart of the automatic synthesis procedure.

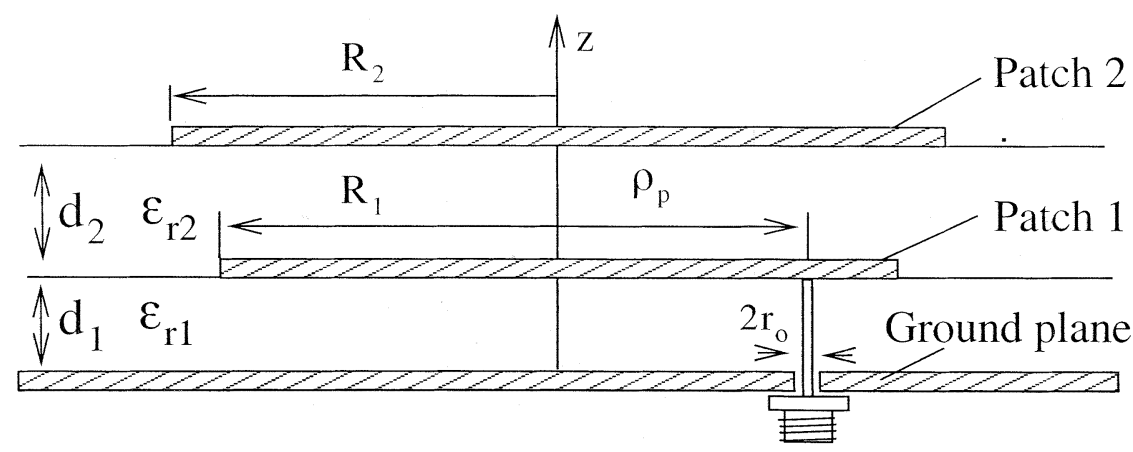

Fig. 2. Cross-sectional view of a probe fed, circular stacked patch antenna.

halted, otherwise, the current parameters and cost are passed to the optimization routine where a new set of parameters is generated. This new set of parameters is checked against the constraints and the cycle continues until the convergence criteria is met.

As in the investigation of [15], the Simplex method [17] is used as the optimization routine due to its stability and reasonable rate of convergence.

\section{A. Antenna Model, Parameters and Constraints}

The patch geometry is presented in Fig. 2. This particular geometry was chosen due to the availability of a fast, accurate simulation tool [18] based on the work of [19] and is the same general structure analyzed in [15]. The antenna consists of two circular conductors with radii $R_{1}$ and $R_{2}$ etched on substrates of dielectric constant $\epsilon_{r 1}$ and $\epsilon_{r 2}$ and thicknesses $d_{1}$ and $d_{2}$ respectively. Substrate 1 is backed with a ground plane. The lower patch is driven by a coaxial feed of radius $r_{0}$, located at a radius of $\rho_{p}$. This feed is attached to a connector through an opening in the ground plane.

There are thus eight variables for optimization $R_{1}, R_{2}, \epsilon_{r 1}$, $\epsilon_{r 2}, d_{1}, d_{2}, r_{0}$, and $\rho_{p}$. As mentioned previously, we wish to examine the dependence of antenna bandwidth and surface wave efficiency on the choice of dielectric substrate layers by choosing $\epsilon_{r 1}$ and $\epsilon_{r 2}$ and recording the optimum solution as a function of this choice. Hence, six variables remain for each optimization.
Constraints must be set to ensure the synthesized patch can be realized. Once the desired operating frequency is chosen, the following loose constraints should be enforced

$$
\begin{aligned}
& 0 \leq R_{1}, R_{2}, d_{1}, d_{2} \leq \frac{\lambda_{0}}{2} \\
& 0 \leq \rho_{p} \leq R_{1}-r_{0}
\end{aligned}
$$

where $\lambda_{0}=$ is the free-space wavelength at the centre of the operating band.

A further constraint must be placed on the thicknesses $d_{1}$ and $d_{2}$ as common laminates are only available with certain thicknesses. To account for this we initially allow a continuous choice of $d_{1}$ and $d_{2}$. On finding an optimum, these thicknesses are rounded to an available thickness and then optimization resumes with $d_{1}$ and $d_{2}$ held at these values. The optimizer can then adjust the remaining four parameters to compensate for the thickness rounding. A practical solution near the optimum is thus achieved.

\section{B. Cost Function}

The cost function is a quantified measure of what we actually want from the optimization, that is, we would like a patch antenna operating at a specified frequency with as broad as possible impedance bandwidth, but which maintains acceptable (80\%) surface wave efficiency. A good radiation pattern can be assumed due to the canonical geometry of the structure. 
The challenge with defining a cost function is how to best translate this request into a mathematical expression indicating the worth of a solution. Here, the cost function is calculated by simulating the antenna performance at 10 frequency points equally spaced from $0.75 \lambda_{0}$ to $1.25 \lambda_{0}$ and then performing weighted sum of three distinct quantities representing mean return loss, bandwidth, and in-band surface wave:

$$
C_{t o t}=w_{R L} C_{R L}+w_{B W} C_{B W}+w_{S W E} C_{S W E} .
$$

The following describes each of these cost components in detail.

1) Minimum Mean Return Loss $\left(c_{r}\right)$ : To optimize the bandwidth, there must first be a matched resonance around the frequency of operation. A completely random case is likely to result in an impedance bandwidth of exactly zero due to the absence of any matched points. Nearby solutions are also likely to have zero bandwidth and thus the bandwidth alone supplies insufficient information for the optimization routine to improve the patch. The optimizer must thus try initially to form an impedance matched resonance. Numerically, this is achieved by minimizing a weighted average of the return loss of all frequency points. Mathematically this can be written

$$
C_{R L}=\frac{1}{N_{f}}\left[\sum_{i=1}^{N_{f}}\left(W\left(f_{i}\right) S\left(\mathbf{x}, f_{i}\right)\right)^{p}\right]^{1 / p}
$$

where $N_{f}$ is the number of frequency points $\left(N_{f}=10\right), f_{i}$ is the frequency at point $i, \mathrm{x}$ is the vector of patch parameters and $S_{i}\left(\mathbf{x}, f_{i}\right)$ is the calculated return loss. The order $(p)$ used was set high $(p=8)$ and the weights $W\left(f_{i}\right)$ were normalized to $10 / N_{f}$ to ensure minimal differentiation between solutions giving $10 \mathrm{~dB}$ return loss or better, but a rapidly increasing penalty for points outside this bandwidth requirement. The weights also favor well matched points closer to the specified operating frequency to encourage the band to be centered near this frequency.

2) Maximum Impedance Bandwidth: Once a resonance is achieved, the actual bandwidth may be optimized. At each iteration, an attempt is made to calculate the $10-\mathrm{dB}$ return loss bandwidth. If it is zero, the cost is also set to zero, otherwise, the cost becomes the reciprocal of the bandwidth value. Mathematically this can be written

$$
C_{B W}= \begin{cases}\frac{1}{B W} & : B W \neq 0 \\ 0 & : B W=0\end{cases}
$$

where BW represents the 10-dB return loss bandwidth.

3) Constrained In-Band Surface Wave Efficiency: The final component of the cost function attempts to ensure efficient solutions are found. To do this, the surface wave efficiency is calculated using the full wave model. Frequency points within the 10 $\mathrm{dB}$ return loss band, with surface wave efficiency $<80 \%$ incur a significant penalty. Thus, the final component of the cost function is

$$
C_{S W E}= \begin{cases}B G & : S W E\left(f_{i}\right)<=80 \%, f_{i} \in B W \\ 0 & : \text { otherwise }\end{cases}
$$

where $B G=10^{10}$ is a dominating penalty.
4) Component Weights: Careful attention must be paid to the weighting of each of the components of the cost function such that the distinct goals that they represent are given the desired priority. The surface wave efficiency is weight $\left(w_{S W E}\right)$ is simply unity as the large penalty will certainly dominate all other cost components. The bandwidth weighting $\left(w_{B W}\right)$ is made 10 times larger than the weight for the average return loss $\left(w_{R L}\right)$ so that bandwidth optimization dominates once a matched resonance is found. In the absence of a matched resonance, only the return loss cost $\left(C_{R L}\right)$ is nonzero and thus $\left(W_{R L}\right)$ may be set arbitrarily to unity.

\section{OPTIMIZATION TRENDS}

Having developed a suitable automatic algorithm, it was possible to begin to explore the design trends and identify the optimal material combinations for broad-band, high-efficiency probe-fed stacked patch antennas. Before presenting this investigation, it will be useful to summarize and validate some previously reported design trends, observations, and "rules of thumb."

First, the dielectric constant of the upper layer must be close to unity to ensure high surface wave efficiency levels, greater than $80 \%$ [2]. Alternatively, if the upper and lower dielectrics are the same and greater than $2.0\left(\epsilon_{r 2}=\epsilon_{r 1}>2\right)$, impedance bandwidths on the order of $45-50 \%$ can be readily achieved; however, the surface wave efficiencies of these configurations are very poor, less than $40 \%$ across the entire 10 -dB return loss bandwidth. For cases when the top layer has a higher dielectric constant than the bottom layer $\left(\epsilon_{r 2}>\epsilon_{r 1}\right)$, only narrow impedance bandwidths can be achieved $(<10 \%)$ as it is extremely difficult to obtain a weakly coupled mutual resonance between the patches. This finding is consistent with that presented in [2] and can be attributed to that the excited currents on each radiator are too strongly coupled. Cases where both dielectric constants are set to approximately $1\left(\epsilon_{r 2}=\epsilon_{r 1} \approx 1\right)$, yield highly efficient antennas as there is no excited surface wave, however, as has been reported in the past [2] increasing the bandwidth with this material combination is difficult. Once again this can be attributed to the difficulty in arranging weakly coupled mutual resonances.

Keeping these rules of thumb in mind, cases were run for different $\epsilon_{r 1}$ values leaving $\epsilon_{r 2}$ constant at 1.07, the value for hard foam. Values of $\epsilon_{r 1}$ simulated were 2.2, 5.0, 7.5, and from 10 to 40 in steps of 5 . All other parameters in the probe-fed stacked patch configuration were set as variables.

Fig. 3 presents the maximum impedance bandwidth achieved by the optimizer for the stacked patch configuration as a function of the dielectric constant of the lower layer $\epsilon_{r 1}$. Each synthesis required several hours of computation on a $400 \mathrm{MHz}$ Celeron. The open circles represent the optimum bandwidth achieved when the procedure was constrained to produce solutions exhibiting a minimum of $80 \%$ in band surface wave efficiency, the filled circles represent the bandwidths of optimum solutions with this constraint relaxed.

Also shown in Fig. 3 is the actual minimum surface wave efficiency within the $10 \mathrm{~dB}$ return loss band exhibited by each of these optimal bandwidth solutions. Solutions where a minimum 


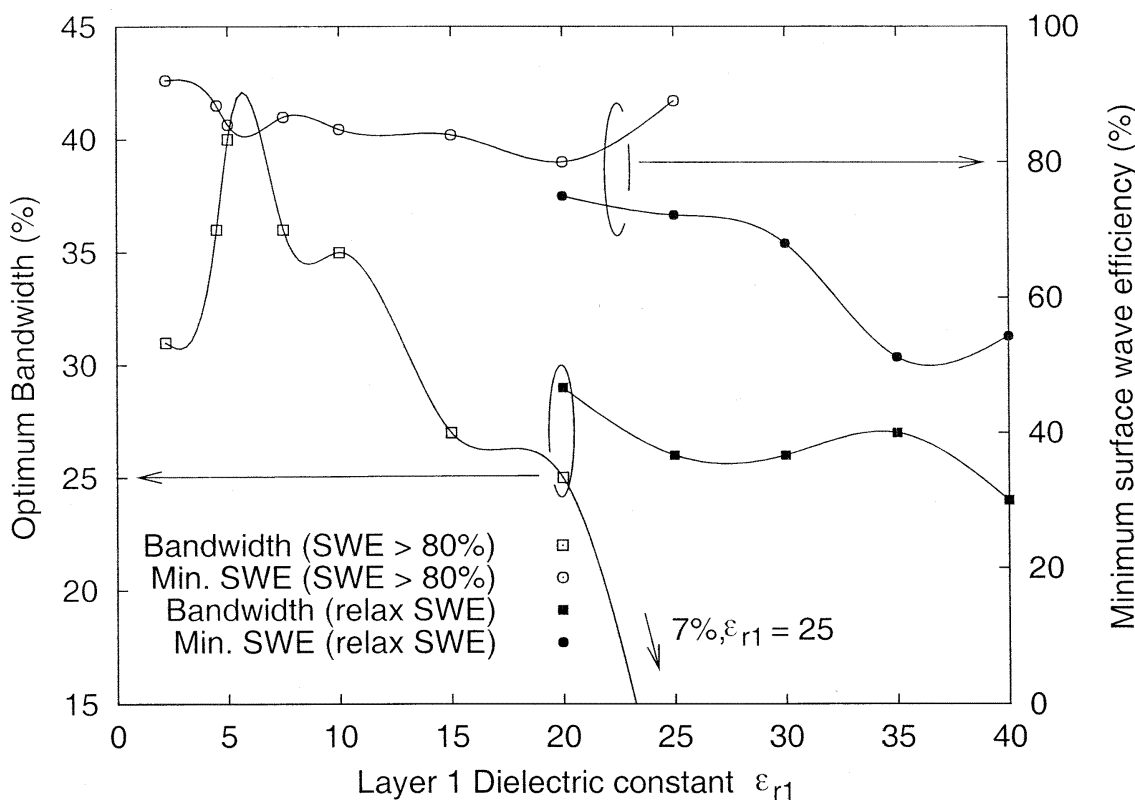

Fig. 3. Optimum bandwidth and minimum, in-band surface wave efficiency as a function of $\epsilon_{r 1}$.

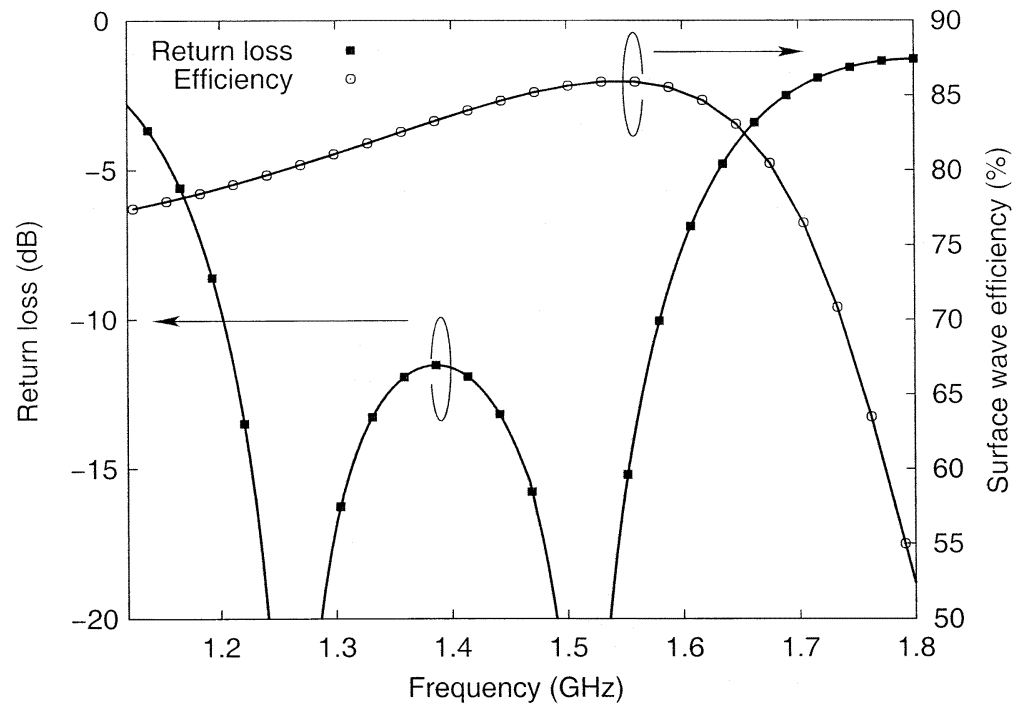

Fig. 4. Frequency response of the return loss and surface wave efficiency for the optimized patch with $\epsilon_{r 1}=15$.

in band surface efficiency $\geq 80 \%$ was enforced are represented by open squares as where the solutions achieved with this condition relaxed are represented by filled squares. Several important trends are evident.

Good impedance bandwidths $(>25 \%)$ and high efficiency $(>80 \%)$ can be achieved for stacked patches with $\epsilon_{r 1} \leq 15$. For $\epsilon_{r 1}>15$, the minimum surface wave efficiency within the 10-dB return loss starts to degrade. In fact, for these cases it was necessary to relax the $80 \%$ constraint on surface wave efficiency to obtain reasonably broad-band solutions at all. Thus, for $\epsilon_{r 1}>20$, although good impedance bandwidth can still be obtained, the minimum surface wave efficiency is significantly reduced, compromising the radiation performance of the antenna.

Fig. 4 presents the return loss and surface wave efficiency frequency response for the optimal case with $\epsilon_{r 1}=15$. Note that the bands in which high efficiency and good impedance bandwidths are achieved overlap, although the maximum efficiency tends to be near the upper frequency edge of the 10-dB return loss bandwidth, similar to the efficient antennas mounted on high dielectric constant material presented in [6] and [7].

Similar frequency responses were calculated for the range of values of $\epsilon_{r 1}$ but are not shown for brevity. It is interesting to note, however, that as $\epsilon_{r 1}$ is reduced, the overlap between the high efficiency band of frequencies and good impedance matching becomes better and the efficiency is maintained over the matched impedance bandwidth declining only slightly with reducing frequency. This implies that the coupling between the 2 radiators improves the surface wave efficiency, and is consistent with the finding shown in [7].

A simplified explanation of this phenomenon is as follows: the surface wave generated from the lower the patch and its as- 
sociated dielectric layers which is normally trapped with the dielectric layers couples to the radiating fields associated with the upper, resonant patch. This explanation is also consistent with the findings in [6], where an oversized resonant ring was used to couple to the surface wave mode, thereby reducing its effect on the efficiency of the antenna.

The trend in Fig. 3 shows that despite this coupling of the surface wave mode to the upper patch, as the dielectric constant of the lower layer is increased the efficiency starts to degrade. This can be explained by having a closer look at the parameter values used to give the solutions in Fig. 3. As $\epsilon_{r 1}$ is increased, good impedance bandwidth and efficiency occurs when the height $d_{1}$ is decreased. Although intuitively, using well-established trends for single layer patches (see for example [11]), the lowering of the height should to an extent counter the increase of $\epsilon_{r 1}$ and therefore the overall contribution to the surface wave may decrease, there is an important effect that needs to be pointed out. As $d_{1}$ decreases, obviously the height of the probe decreases and so the effect of the discontinuity associated with the probe feed becomes less pronounced [19]. It has been shown in the past that surface waves couple well to discontinuities and that the presence of possible discontinuities can determine whether the power is trapped within a surface wave or radiated [20]. By reducing the height of $d_{1}$ the discontinuity associated with the feed pin decreases and therefore the amount of power coupled to the surface wave decreases. Further investigation into this phenomenon is currently being undertaken.

From Fig. 3 there appears to be an optimum solution for a probe-fed stacked patch antenna where the impedance bandwidth and the minimum surface wave efficiency are both high. This occurs when $\epsilon_{r 1}=5$. For this case the theoretical $10 \mathrm{~dB}$ return loss approaches $40 \%$ and the minimum surface wave efficiency is approximately $90 \%$. This return loss bandwidth is significantly larger than previously reported probe-fed stacked patch solutions [2], [7], approaching values obtained for large slot aperture coupled patches configuration [11] and small slot-sized aperture coupled stacked patches [1]. The probe-fed stacked patch solution has the advantage of reduced backward directed radiation compared to the aperture solutions. It should be noted that further enhancement in bandwidth performance may be achieved using a triple stacked patch configuration [21]; however, this was not investigated here.

\section{RESUlTS AND DISCUSSION}

Due to the unavailability of commercial microwave laminates with a dielectric constant of 5.0, an optimized probe-fed stacked patch solution was sought utilizing standard PCB (FR4, $\epsilon_{r}=$ $4.5)$ as the bottom layer. This material can be somewhat lossy for frequencies above $2 \mathrm{GHz}$ so an investigation centered at approximately $1.4 \mathrm{GHz}$ was conducted. To ensure a solution could be realized, the thickness of the bottom layer $d_{1}$ was limited to multiples of $1.59 \mathrm{~mm}$ and the upper foam layer $d_{2}$ to multiple combinations of 3,5 or $6 \mathrm{~mm}$ thick laminates.

Fig. 5 shows the predicted input impedance response of the optimized stacked patch (refer to the figure caption for parameters). The $10 \mathrm{~dB}$ return loss bandwidth is approximately $37 \%$, approaching that achieved for the optimum results presented in

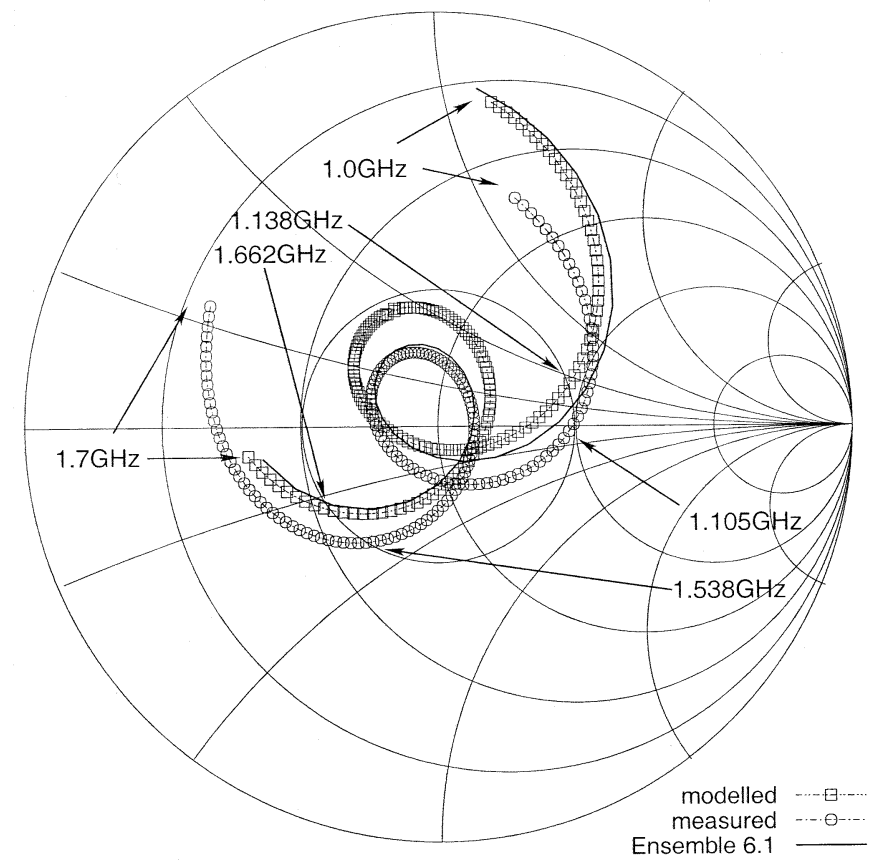

Fig. 5. Impedance response of the optimized stacked patch with parameters: $R_{1}=29.4, R_{2}=43.8, d_{1}=10.7, d_{2}=17.0, r_{0}=3.3$, and $\rho_{p}=$ $20.4 \mathrm{~mm}$, with $\epsilon_{r 1}=1.07$ and $\epsilon_{r 2}=4.5$. The measured and optimizer simulated results are represented by squares and circles, respectively, with the solid line depicting simulated performance from Ensemble 6.1 for comparison.

Section III. Fig. 5 also presents the impedance locus for the optimized dimensions predicted by Ensemble 6.1 [22]. For this simulation, the circular patch was divided into 36 segments and thus it is possible that discretization errors may account for the slight discrepancy between the simulated results of Ensemble and the full wave analysis used during optimization.

The proposed antenna was fabricated using standard etching techniques. A thin layer of Duriod 5880 (thickness of 0.254 $\mathrm{mm}$ and a dielectric constant of 2.2) was used to etch the conductor of the top patch located above the layers of foam in the stacked patch arrangement. To realize the feeding pin, a wire with a thickness of $6 \mathrm{~mm}$ and a height of $12 \mathrm{~mm}$ was soldered to the conductor of the bottom patch and to an $\mathrm{N}$-type connector located below the ground plane of the antenna. The measured impedance response of this realized antenna is also presented in Fig. 5. As seen from this figure, there is reasonably good agreement between measurement and predicted results. The measured $10-\mathrm{dB}$ return loss is $33 \%$. The slight reduction in bandwidth (37\% predicted) and center frequency (actually centered at $1.323 \mathrm{GHz}$ ) can be attributed to the following factors: 1) the thin layer of material used to etch the top patch is not included in the predicted results; 2) the uncertainty in the dielectric constants of FR4 and the hard foam; and 3) thin air-gaps between the constructed layers.

A capacitive shift is also evident in the measured response when compared to the theoretical predictions, which may contribute to the reduction in the observed bandwidth. This shift is due to manufacturing difficulties associated with connecting the electrically thick probe feed to conventionally styled connectors. The diameter of the hole in the ground plane to accommodate for the pin connector needs to be large enough such that 


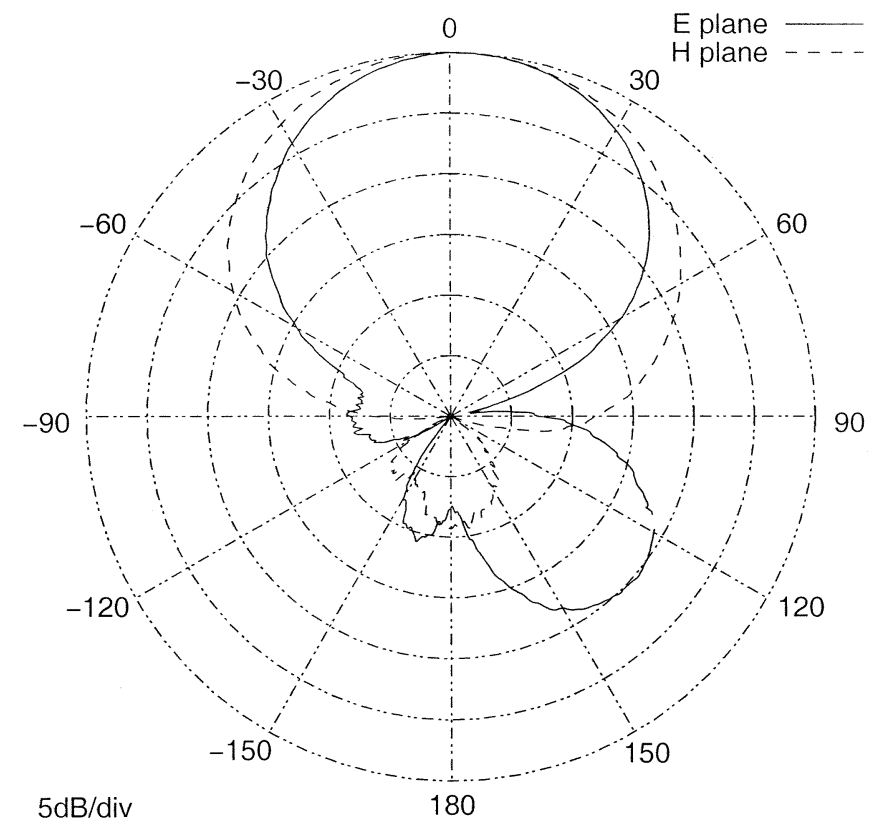

Fig. 6. Measured copolar $\mathrm{E}$ and $\mathrm{H}$ plane radiation patterns for the optimized patch at $1.4 \mathrm{GHz}$.

a $50 \Omega$ transition between the guided medium (a coaxial line) and the bottom of the patch antenna is maintained. From Fig. 5 it is apparent that the hole in the ground plane is slightly undersized.

The measured copolar $\mathrm{E}$ and $\mathrm{H}$ plane radiation patterns of the optimized stacked patch antenna at $1.4 \mathrm{GHz}$ are shown in Fig. 6. The radiation patterns are very similar to the computed patterns (not shown here for the sake for brevity) and are typical for a stacked patch configuration. The ground plane for the antenna was very small, extending only $2 \mathrm{~cm}$ beyond the edge of the top patches. For this reason, there appears to be a slight distortion in the patterns, particularly for the E plane case, in the backward directed plane. The resulting copolar patterns are similar to other highly efficient printed antennas [2], [6], [7]. The measured cross-polarized levels are less than $-20 \mathrm{~dB}$ in the E plane and less than $-12 \mathrm{~dB}$ in the $\mathrm{H}$ plane below the copolar fields. These values compare to theoretical levels of less than $-40 \mathrm{~dB}$ and $-14 \mathrm{~dB}$, respectively. Once again, the finite ground plane contributes to the higher than predicted levels. It is interesting to note that the predicted and measured cross-polar levels are not as low for the case when a higher dielectric constant material is used for the lower layer, as in [7] or in fact even those in [2].

One source for these comparatively high levels could be the relatively thick $(6 \mathrm{~mm})$ feed pin used to obtain the optimally efficient and broad-band solution. As was presented in [23], the probe does contribute to the cross-polarized field, and the thicker the probe, the higher the cross-polar content. From this there is another apparent compromise that must be made in the choice of the optimum solution of a stacked patch antenna: using thicker probes can yield better bandwidths; however, the cross-polarized levels are also increased. A further investigation of this will be presented later.

The theoretical and measured gain of the stacked antenna was $9.0 \mathrm{dBi}$ and $8.0 \pm 0.5 \mathrm{dBi}$ at $1.4 \mathrm{GHz}$, respectively. The discrep- ancy can be resolved by considering the large backlobe in the $\mathrm{H}$ plane and the accuracy of the antenna range $( \pm 0.5 \mathrm{~dB})$. These results verify that efficiency has not been compromised to attain large bandwidth.

\section{CONCLUSION}

This paper has presented the development of an automatic optimization routine that can synthesize broad-band and efficient antenna designs, which can be practically fabricated using readily available materials and techniques. The described optimization tool has been utilized to explore the requirements for highly efficient, broad-band antenna solutions. A design trend relating the choice of dielectric materials to the optimum achievable bandwidth and surface wave efficiency has been identified and the physical processes determining its behavior have been discussed.

A particularly high-performance peak in the design trend was chosen to examine the practical performance of the synthesized solutions. The realized antenna performed close to expectations yielding a $33 \%$ bandwidth at $1.4 \mathrm{GHz}$ while maintaining greater than $80 \%$ surface wave efficiency across the band.

The optimization approach is thus verified as an excellent tool for the investigation of antenna design requirements, and also for the synthesis of very high-performance solutions. To proceed with this research, it would be beneficial to examine a more general class of antenna, such as aperture stacked patches [24] and antennas of less constrained conductor geometry [16]. This development would require the introduction of a more general electromagnetic simulator into the optimization routine. The cost function may also be developed further to more precisely specify the desired trade off between efficiency and bandwidth and may also be extended to include factors such as radiation and polarization performance and also the physical size of the antenna.

\section{ACKNOWLEDGMENT}

The authors would like to acknowledge D. Welch for his insight and technical expertise.

\section{REFERENCES}

[1] F. Croq and D. M. Pozar, "Millimeter wave design of wide-band aperture-coupled stacked microstrip antennas," IEEE Trans. Antennas Propagat., vol. 39, pp. 1770-1776, Dec 1991.

[2] R. B. Waterhouse, "Design of probe-fed stacked patches," IEEE Trans. Antennas Propagat., vol. 47, pp. 1780-1784, Dec 1999.

[3] J.-S. Kuo and K.-L. Wong, "A dual-frequency l-shaped patch antenna," Microwave Opt. Technol. Lett., vol. 27, pp. 177-179, Nov 2000.

[4] G. Kumar and K. C. Gupta, "Non radiating edges and four edges gap-coupled multiple resonator broad-band microstrip antennas," IEEE Trans. Antennas Prop., vol. 33, pp. 173-178, Feb 1985.

[5] D. R. Jackson, T. J. Williams, A. K. Bhattacharyya, R. L. Smith, S. J. Buchheit, and S. A. Long, "Microstrip patch designs that do not excite surface waves," IEEE Trans. Antennas Propagat., vol. 41, pp. 1026-1037, Aug 1993.

[6] D. M. Kokotoff, R. B. Waterhouse, C. R. Birtcher, and J. T. Aberle, "Annular ring coupled circular patch with enhanced performance," Electron. Lett., vol. 33, pp. 2000-2001, Nov 1997.

[7] R. B. Waterhouse, "Stacked patches using high and low dielectric constant material combinations," IEEE Trans. Antennas Propagat., vol. 47, pp. 1767-1771, Dec 1999. 
[8] T. K. Lo, C. O. Ho, Y. Hwang, E. K. W. Lam, and B. Lee, "Miniature aperture-coupled microstrip antenna of very high permittivity," Electron. Lett., vol. 9-10, pp. 1026-1037, Jan 1997.

[9] R. B. Waterhouse, S. D. Targonski, and D. M. Kokotoff, "Design an performance of small printed antennas," IEEE Trans. Antennas Propagat., vol. 46, pp. 1629-1633, Nov 1998.

[10] K. L. Wong and Y. F. Lin, "Small broadband rectangular microstrip antenna with chip-resistor loading," Electron. Lett., vol. 33, pp. 1593-1594, Sept 1997.

[11] J.-F. Zurcher and F. E. Gardiol, Broadband Patch Antennas. Boston, MA: Artech House, 1995.

[12] Internal Antennas for Handset Applications. [Online]. Available: http://www.centurion.com/mp4.htm

[13] D. M. Kokotoff, R. B. Waterhouse, and J. T. Aberle, "Figure of merit for the design of microstrip patch antennas," in Proc. IEEE Antenna Propagat. Society Int. Symp., Orlando, FL, July 1999, pp. 2478-2481.

[14] D. M. Pozar and D. H. Schaubert, Microstrip Antennas. Piscataway, NJ: IEEE Press, 1995.

[15] M. Lech, A. Mitchell, and R. B. Waterhouse, "Optimization of broadband microstrip patch antennas," in Proc. 2000 Asia Pacific Microwave Conf., Sydney, Astralia, Dec 2000, pp. 711-714.

[16] J. M. Johnson and Y. Rahmat-Samii, "Genetic algorithms and method of moments (GA/MOM) for the design of integrated antennas," IEEE Trans. Antennas Propagat., vol. 47, pp. 1606-1614, Oct. 1999.

[17] W. H. Press, S. A. Teukolsky, W. T. Vetterling, and B. P. Flannery, $N u$ merical Recipes in C: The Art of Scientific Computing. Cambridge, U.K.: Cambridge Univ. Press, 1996.

[18] D. M. Kokotoff, R. B. Waterhouse, and J. T. Aberle, "On the use of attachment modes in the analysis of printed antennas," in Proc. Progress in Electromagnetic Res. Symp. (PIERS), Nantes, France, July 1998, pp. 13-17.

[19] J. T. Aberle and D. M. Pozar, "Accurate and versatile solutions for probe fed microstrip patch antennas and arrays," Electromagn., vol. 11, no. 1, pp. 1-19, Jan 1991

[20] R. B. Waterhouse, "The use of shorting pins to improve the scanning range of probe-fed microstrip patch phased arrays," IEEE Trans. Antennas Propagat., vol. 44, pp. 302-309, Mar. 1996.

[21] W. Rowe and R. B. Waterhouse, "Broadband microstrip patch antennas for MMIC's," Electron. Lett., vol. 36, pp. 597-599, Apr. 2000.

[22] Ensemble 6.1, Software, Ansoft, 1999.

[23] R. B. Waterhouse, "Improving the scan performance of probe-fed microstrip patch arrays," IEEE Trans. Antennas Propagat., vol. 43, pp. 705-712, July 1995

[24] S. D. Targonski, R. B. Waterhouse, and D. M. Pozar, "Design of wideband aperture-stacked patch microstrip antennas," IEEE Trans. Antennas Propagat., vol. 46, pp. 1245-1251, Sept. 1998.

Arnan Mitchell was born in Dublin, Ireland, on February 20, 1973. He received the B.Tech. degree (Hons.) in optoelectronics from Macquarie University, NSW, Australia, in 1993 and the Ph.D. degree from the Royal Melbourne Institute of Technology (RMIT) University, Melbourne, Australia.

He is currently an Australian Photonics CRC Research Fellow, RMIT University, where he is investigating broadband and specialized integrated optical modulators and RF photonic components for communications and signal processing applications. His research interests include numerical methods and optimization techniques and their application to RF-photonic devices.
Margaret Lech received the M.Sc. degree in applied physics from Maria CurieSklodowska University, Poland, in 1982 and the Ph.D. degree in electrical engineering from The Melbourne University, Melbourne, Australia, in 1993.

From 1993 to 1995, she was a Research Scientist with Monash University, Melbourne, where she worked on the development and optimization of mathematical models for automatic close-range photogrammetry systems. From 1995 to 1997, she was with The Bionic Ear Institute, Melbourne, working in the areas of speech processing and psychophysical characteristics of speech perception. Since 1997, she has been with The Royal Melbourne Institute of Technology (RMIT) University, Melbourne, Australia, where she is currently a Lecturer in electrical engineering. Her research interests include digital signal processing, system modeling, and optimization.

David M. Kokotoff received the B.S.E.E. degree from Lafayette College, Easton, PA, in 1985, the M.S.E.C.E. degree from the University of Massachusetts, Amherst, in 1987, and the Ph.D. degree from Arizona State University, Tempe, in 1995.

From 1987 to 1992, he was he was a Member of the Advanced Antenna Development Group with Atlantic Aerospace Electronics Corporation, Greenbelt, MD. From 1996 to 1999, he was a Lecturer with the Department of Communication and Electronic Engineering, Royal Melbourne Institute of Technology (RMIT) University, Melbourne, Australia. In 1999, he became an Antenna Design Engineer with Gabriel Electronics Inc., Scarborough, ME, focusing on antennas for wireless broad-band applications. His research interests include applied electromagnetics emphasizing computer-aided design of antennas, microwave, and optical devices. He has published more than 25 journal and conference papers and holds one patent.

Rod B. Waterhouse (S'90-M'94-SM'01) received the B.E. (Hons.) M.Eng.Sc., and Ph.D. degrees from the University of Queensland, Australia, in 1987,1990 , and 1994 , respectively.

In 1994, he joined the School of Electrical and Computer Engineering, Royal Melbourne Institute of Technology (RMIT) University. From 2000 to 2001, while on sabbatical, he was a Visiting Professor with the Department of Electrical and Computer Engineering, University of California, Los Angeles, and then a Visiting Researcher with the Photonics Technology Branch at the Naval Research Laboratories. Washington, DC. In June 2001, he took a leave of absence from RMIT and joined Dorsal Networks, MD. His research interests include printed antennas, optically distributed wireless systems, photonic devices and optical systems. He has published more than 150 papers and has three patents

Dr Waterhouse chaired the IEEE Victorian MTTS/APS Chapter from 1998 to 2001 